\title{
Dynamic Change in the Prominent Vessel Sign according to the Perfusion Status
}

\author{
Sang Hee Ha* (D), Ho Geol Woo ${ }^{\dagger}$ (D), Hyun Ho Kim (D), Bum Joon Kim (D) \\ Department of Neurology, Kyung Hee University Hospital ${ }^{\star}$, Seoul; Department of Neurology, Ewha University Seoul Hospital ${ }^{\dagger}$, Seoul, Korea
}

An 87-year old man with hypertension, diabetes, and renal failure was admitted with aphasia and right-sided weakness. Susceptibility-weighted imaging (SWI) revealed prominent vessel sign (PVS) without an acute lesion, with left carotid artery occlusion and decreased perfusion of the left hemisphere. The blood pressure increased from $90 / 60 \mathrm{mmHg}$ to $160 / 80 \mathrm{mmHg}$ after hypertensive treatment and the neurological deficits recovered completely. Five hours later, the PVS decreased on follow-up SWI. The PVS dynamically changes according to the perfusion status; therefore, it may be potentially beneficial to evaluate the perfusion status after treatment, especially in patients in whom contrast agent use is contraindicated.

J Neurosonol Neuroimag 2019;11(2):166-16S

Key Words: Magnetic resonance image; Prominent vessel sign; Induced hypertension; Follow-up studies
Received: October 28, 2018

Revised: November 27, 2019

Accepted: November 30, 2019

Address for correspondence: Bum Joon Kim

Department of Neurology, Kyung Hee University Hospital, Kyung Hee University College of Medicine, 23 Kyungheedae-ro, Dongdaemun-gu, Seoul 02447, Korea

Tel: $+82-2-958-8499$

Fax: $+82-2-958-8490$

E-mail: medicj8o@hanmail. net
Occlusion of the internal carotid artery (ICA) can cause ischemia through various mechanisms, primarily through artery-to-artery embolisms from the stump, but also due to a hemodynamic mechanism. When the patient is in a hypotensive condition, the collateral supply may be insufficient. Susceptibility-weighted imaging (SWI) is sensitive for detecting dilated vessels, which can be observed in the area of hypoperfusion (prominent vessel sign, PVS). Here, we report a patient who initially showed the PVS that dynamically decreased a few hours after induced hypertension with associated neurological recovery.

\section{CASE REPORT}

An 87-year old man with hypertension, diabetes, and renal failure was admitted with aphasia and right-sided weakness (Motor Research Council grade 2 in both arm and leg). The neurological symptoms were fluctuating upon admission. Previously, the patient was under regular medication with an angiotensin receptor blocker and metformin. Five days before the visit, the patient experienced severe diarrhea for 3 days without fever. The initial blood pressure was $90 / 60 \mathrm{mmHg}$ and the heart rate was 102 beats/min. Serologic analysis revealed elevated blood urea nitrogen (31 mg/dL) and creatinine (1.64 mg/dL) levels. SWI revealed PVS without an acute lesion, with left carotid artery occlusion and decreased perfusion of the left hemisphere (Fig. 1A-D).

Induced hypertension treatment was administered with continuous intravenous phenylephrine infusion $(1.6 \mathrm{mg} / \mathrm{h})$. After 1 hour, the blood pressure had risen to $160 / 80 \mathrm{mmHg}$ and the neurological deficits had fully recovered. Four hours later, the PVS had dynamically decreased as indicated by follow-up SWI (Fig. 1E). Furthermore, surgical bypass surgery was not considered due to the patient's age, and blood pressure medications were discontinued, aiming to maintain a systolic blood pressure above $140 \mathrm{mmHg}$. No further worsening of neurological deficits was detected for 3 months after the event. 

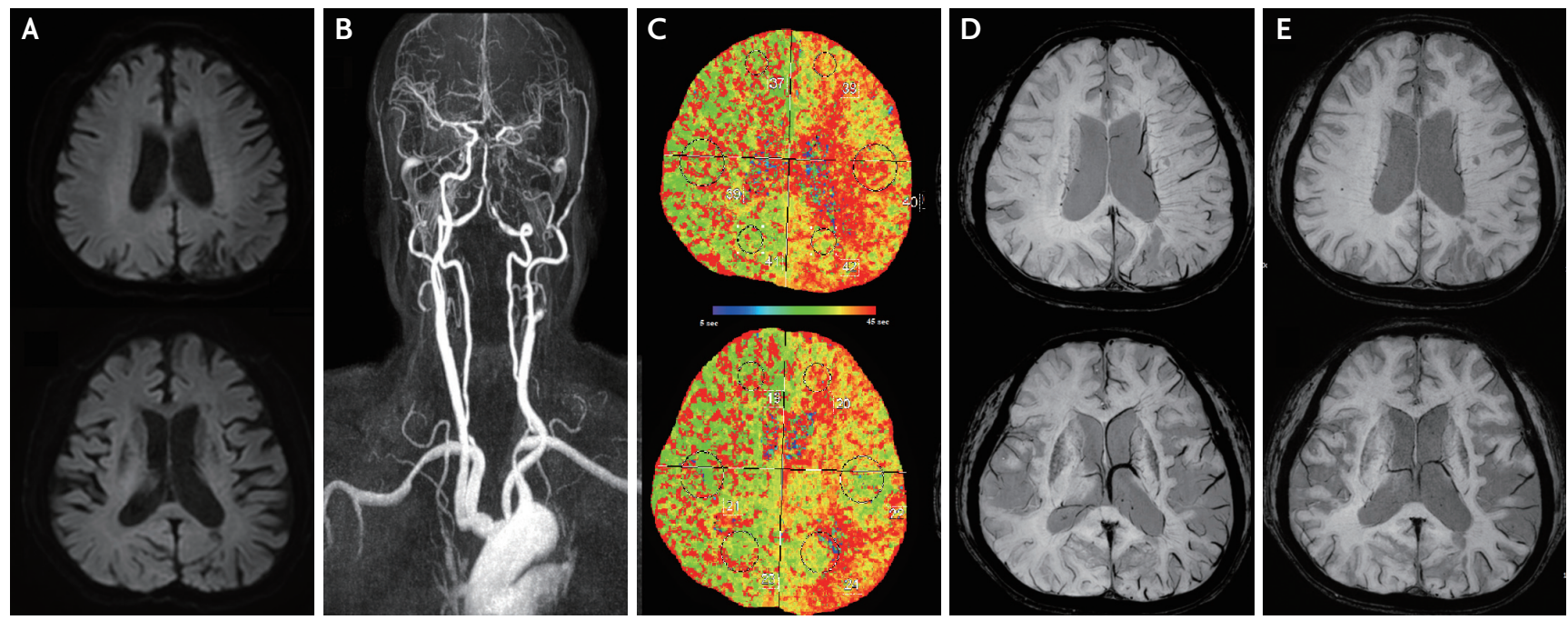

FIG. 1. (A-D) Initial and (E) follow-up images. (A) Axial diffusion-weighted image and (B) angiography image revealing collateral flow (C) with a prolonged mean transit time (computed tomography perfusion image). (D) Initial and (E) follow up (5 hours later), susceptibility-weighted imaging scans showing dynamically reduced prominent vessel sign in the cortical, medullary, and thalamostriate vein in the left hemisphere.

\section{DISCUSSION}

The PVS was developed due to the increased concentration of deoxyhemoglobin inside the vein where the oxygen extraction ratio was increased. Therefore, the PVS was thought to represent the ischemic penumbra. The PVS predicted infarct growth, early neurological deterioration, and poor outcome. ${ }^{1,2}$ However, there is a lack of data showing how the PVS present on SWI may change dynamically.

Induced hypertension increases perfusion. The treatment is effective and feasible for those who may not receive reperfusion treatment. Several studies have reported promising results of the treatment. ${ }^{3,4}$ In our patient with ICA occlusion, induced hypertension was effective. The treatment effect was not only reflected in the neurological improvement, but also objectively in the dynamic change in the PVS in the short-term follow-up analysis performed within a few hours.

The perfusion status is usually determined using computed tomography or magnetic resonance perfusion imaging. ${ }^{5}$ However, a considerable amount of the contrast agent is required in both studies. Using contrast repeatedly within a day may have a potential adverse effect on the renal function and should be avoided for patients with poor renal function. ${ }^{6}$ However, a contrast agent is not required in SWI; the scan time is relatively short, and the PVS changes dynamically according to the perfusion status after a few hours. Therefore, our case findings suggest that the PVS may be potentially beneficial for evaluating the perfusion status, especially after treatment inducing increased perfusion to the cerebral hemisphere, especially in patients with poor renal function.

\section{Acknowledgments}

This work was supported by the National Research Foundation of Korea (NRF) grant funded by the Korea government (MSIT) (No. 2017R1CiB5017843).

\section{Conflicts of Interest}

No potential conflicts of interest relevant to this article was reported.

\section{REFERENCES}

1. Chen CY, Chen CI, Tsai FY, Tsai PH, Chan WP. Prominent vessel sign on susceptibility-weighted imaging in acute stroke: prediction of infarct growth and clinical outcome. PLoS One. 2015;10:e0131118.

2. Wang Y, Shi T, Chen B, Lin G, Xu Y, Geng Y. Prominent hypointense vessel sign on susceptibility-weighted imaging is associated with clinical outcome in acute ischaemic stroke. 
Eur Neurol. 2018;79:231-239.

3. Lee MH, Kim JG, Jeon SB, Kang DW, Kwon SU, Kim JS. Pharmacologically induced hypertension therapy for acute stroke patients. J Stroke. 2019;21:228-230.

4. Kim HJ, Kang DW. Induced hypertensive therapy in an acute ischemic stroke patient with early neurological deterioration. J Clin Neurol. 2007;3:187-191.
5. Chalela JA, Dunn B, Todd JW, Warach S. Induced hypertension improves cerebral blood flow in acute ischemic stroke. Neurology. 2005;64:1979.

6. Mehran R, Nikolsky E. Contrast-induced nephropathy: definition, epidemiology, and patients at risk. Kidney Int Suppl. 2006;(100):S11-S15. 